\title{
Reliability Increase of Base Heating Prediction and Thermal Protection Methodology for Launch Vehicle Upper-Stage
}

\author{
Yiying Bao ${ }^{\mathrm{a}, \mathrm{b}}$, Yifu Ding ${ }^{\mathrm{a}, \mathrm{b}}$, Pingyang Wang ${ }^{\mathrm{b}}$, and Zhenhua Liu ${ }^{\mathrm{b}}$,* \\ ${ }^{a}$ Shanghai Aerospace System Engineering, Shanghai, 201108, China \\ ${ }^{b}$ Shanghai Jiaotong University, Shanghai, 200240, China
}

\begin{abstract}
During spacecraft missions in some countries all over the world, a number of failures have been observed that were due to the lower reliability of thermal protection of launch vehicles, so the reliability of base heating prediction and thermal protection is very important for successful emissions. The thermal protection of launch vehicles is usually carried out according to the base heating prediction. The base heating environment is a complicated combination of exhaust plume radiation, reversed plume flow convective heating, and engine nozzle radiation. In this paper, the numerical analysis of base heating is performed to improve the precision of the predictive analysis, and then the flow is modeled using a two-dimensional, Reynolds averaged, and compressible Naive-Stokes equations and DSMC for a chemically frozen mixture gas. After that, the radiation is modeled by using RMC. On the basis of this elaborate base heating prediction, the weaknesses of the thermal protection can be found and the elaborate thermal protection can be realized, so the reliability of base heating prediction and thermal protection methodology can be increased.
\end{abstract}

Keywords: reliability increase; upper-stage; plume heating; predictive analysis; thermal protection

(Submitted on October 9, 2018; Revised on November 15, 2018; Accepted on December 20, 2018)

(C) 2019 Totem Publisher, Inc. All rights reserved.

\section{Introduction}

According to the diversity of satellite-launching demands, different kinds of upper-stages of launch vehicles have been developed. Some upper-stages can be used to carry several satellites into space orbits of different altitudes, while others can be used to deploy several satellites at different orbit positions. Nearly all these upper-stages of launch vehicles use great thrust force engines for navigation and gesture controlling, and these engines are usually started up several times in the space orbit. During the space working time of the engine, the upper-stage is exposed to a space vacuum and cold environment, and its after-body is heated by exhaust plume and non-regenerative cooling nozzles. The exhaust plume heating has two forms: high-temperature gas radiation and reversed flow convection.

Regarding the numerical computation of base heating environments, it is well known that the continuum Navier-Stokes equations can provide an accurate physical model for the flow of great thrust force engines working on the ground and in the powered ascent, and direct simulation Monte Carlo (DSMC) has been used frequently for the flow simulation of minor thrust force engines working in space orbit. In recent decades, DSMC has been a mature technique for rarefied flow simulation.

In 2004, Wang et al. of Shanghai Jiaotong University simulated the plume flow field of rocket engines in a vacuum environment by DSMC with the variable soft ball model (VSS) [1]. In 2009, Li et al. of the National University of Defence Technology used the model proposed by Gallis to solve the force and heat interaction between gas molecules and solid particles, demonstrating the mechanism of momentum and energy transmission in the progress of the interaction [2]. In 2011, Tang et al. of Beijing University of Aeronautics and Astronautics simulated the plume flow field in a vacuum environment using the decoupling N-S/DSMC method [3]. In 2015, Li et al. adopted the DSMC method to simulate the

\footnotetext{
* Corresponding Author.

E-mail address: byywin@163.com
} 
plume flow field of high altitude solid rocket motors [4]. In 2017, Ding et al. of Shanghai Jiaotong University used the DSMC method to simulate the plume flow field of high altitude solid rocket motors, taking into account the phase transformation process of solid particles [5].

The study of the thermal environment at the bottom of rockets mainly involves the acquisition of total heat flow, including convection heat flow and radiation heat flow. In the uncoupled algorithm, the convection data are obtained by analyzing the plume field of the rocket motor, and the radiation heat flow data are obtained by analysing the gas radiation transmission.

In 2004, Dong et al. of Harbin Institute of Technology adopted the RMC method instead of the traditional DSMC method to simulate heat radiation transmission in participatory media, and they achieved good results [6]. In 2005, Qi et al. of Shanghai Jiaotong University adopted the RMC method to simulate the infrared radiation characteristics of lobed nozzle/mixer plume [7]. In 2009, Yang et al. of Shanghai Jiaotong University adopted the RMC method to simulate the base heating environment of the secondary-stage of one launcher and obtained the changing trends of bottom heat flow with height [8]. In 2012, Li et al. of Nanjing University of Science and Technology investigated the effects of the alumina particle phase change process and a small amount of carbon soot on the radiation field using the RMC method [9]. In 2016, Sun et al. adopted the DSMC method to simulate the flow field of one rocket's third-stage motor, and the RMC method was adopted to simulate the radiation heat flow received at different locations and compare the experiment results [10].

Although there are many computational studies on flow field and radiation, it is seldom individually used for great thrust force engines working in space orbit because of the limitation of computing efficiency and the memory usage.

In this paper, to increase the reliability of base heating prediction and thermal protection methodology, the numerical analysis of base heating for upper-stage of launch vehicles with great thrust force engines working in a vacuum space is performed, the N-S equations and DSMC are coupled for this continuum/rarefied flow simulation, and the RMC method is used for radiation simulation. The results of the convective heat flux of plume, radiative heat flux of plume, and nozzle are given respectively. The total exterior heat flux conditions of the different typical positions at the base of the upper-stage are obtained for designing the elaborate thermal protection system. The verification of this method is carried out by the comparison of the computed data and the measured data of the flight third-stage of one launch vehicle.

\section{Modeling and Numerical Method}

\subsection{Convective Heating of Plume}

In this paper, the plume includes the continuum flow region, slip flow region, transition flow region, and free molecule flow region at the same time. For different flow regions, different models are in application. In the continuum flow region, the frequency of collision between different molecules is far greater than the frequency of collision between molecules and walls; the gas motion can be described by N-S equations, the Fourier heat transfer equation, and the Fick mass diffusion equation. In the slip flow region, the continuous medium is still in domination, so the above equations are still in application, but the phenomena of velocity slip, temperature jump, and heat slip can be observed. In the transition flow region, the molecular free path has the same magnitude as the characteristic dimension of flow, and the collision between molecules and walls should be considered as the collision between different molecules. Because the behaviour of incoming molecules is relevant to the walls and the reflected molecules may change the motion direction of incoming flow and the quantities of incoming molecules to the wall, the continuous medium hypothesis is not proper, and the Boltzmann equation is more suitable to describe the rarefied flow motion. In the free molecule flow region, the molecular free path is far greater than the characteristic dimension of flow, and only the collision between molecules and walls should be considered while the collision between different molecules can be ignored. The DSMC method was put forward by Bird in the 1970s to solve the rarefied gas flow dynamics problems, and it has been popularly used since the 1980s. It was reported that Boyd, Campbell, and Nelson used the DSMC method for numerical simulation of rarefied expansion flow. To verify the application of DSMC in space plume, Boyd et al. also compared the computed data and the measured data of space plume in 1991 and 1992 [11-12]. Oh and Hastings used the quasi-neutrality cell particles-direct simulation Monte Carlo (PIC-DSMC) method to simulate the space plume of the Holtz propeller of flight vehicles [13]. Gimelshein et al. put forward a discrete model of multi-atom molecules' energy transport using the rotation/vibration model and translation model. George and Boyd used a coupled method of CFD-DSMC for the simulation of plume in a vacuum space to save the computing time [14].

In this paper, the altitude of the new upper-stage working-orbit in the space is $200-1000 \mathrm{~km}$. Nitrogen tetroxide $\left(\mathrm{N}_{2} \mathrm{O}_{4}\right)$ and dimethylhydrazine (UDMH) are used as propellants. The nozzle is cooled by the regenerative system and the radiative way, and the temperature of the radiative-cooling nozzle body is $1450 \mathrm{~K}$. The outlet combustion gas is a mixture of $\mathrm{H}_{2} \mathrm{O}$, 
$\mathrm{CO}_{2}, \mathrm{CO}, \mathrm{N}_{2}$, and $\mathrm{H}_{2}$. The propulsive force is $5000 \mathrm{~N}$. Because of the high rate of propellant flow, the plume in the adjacent region of the nozzle outlet is a continuum flow where the density and the pressure is higher; however, the plume far away from the nozzle and the plume in the reversed flow region is rarefied flow because of the high vacuum, so the reasonable technical route is to make different models for different kinds of flow. In this paper, the scheme is to solve the N-S equation in a finite difference way, coupled with DSMC of gaseous mixtures to obtain the convective heat flux of the plume. The three steps are taken as follows:

Step 1 In the continuum flow region, the plume is modelled in the FLUENT software for the Navier-Stokes approach. By solving the N-S equation in a finite difference way, the numerical simulation of the nozzle inlet flow and the continuum flow of the plume can be realized.

In this study, the new upper-stage uses the swing singe-nozzle engine with great thrust force, the swing angle is \pm 25 , and the hypothesis is that the plume is circumferential homogeneous distribution, so a two-dimensional symmetric model of the inner moulding surface of the nozzle is made, as shown in Figure 1. The symmetry axis of the nozzle is set to be the axial boundary, and the throat cross section of the nozzle is set to be the pressure inlet boundary of the flow. The total temperature, the total pressure, and the static temperature of the throat cross section are given as fixed numerical values. The length of the flow computing domain is 25 times the outlet radius of the nozzle, and the environment is set to be the pressure outlet boundary. In this study, the pressure is given according to an altitude of $200 \mathrm{~km}$. The gas flow in the nozzle is assumed to be one-dimensional thermal insulation isentropic flow, the flow is homogeneous compositing and steady, and the viscous property can be ignored. The mole percent of the gas component is constant, that is, the flow is assumed to be chemical frozen flow. Because the plume changes severely in the centrum, the mesh adjacent to the axis of the nozzle is in subdivision, as shown in Figure 2. The flow is compressible turbulent flow, so the two-equation k- $\varepsilon$ model using the explicit coupled algorithm is chosen.

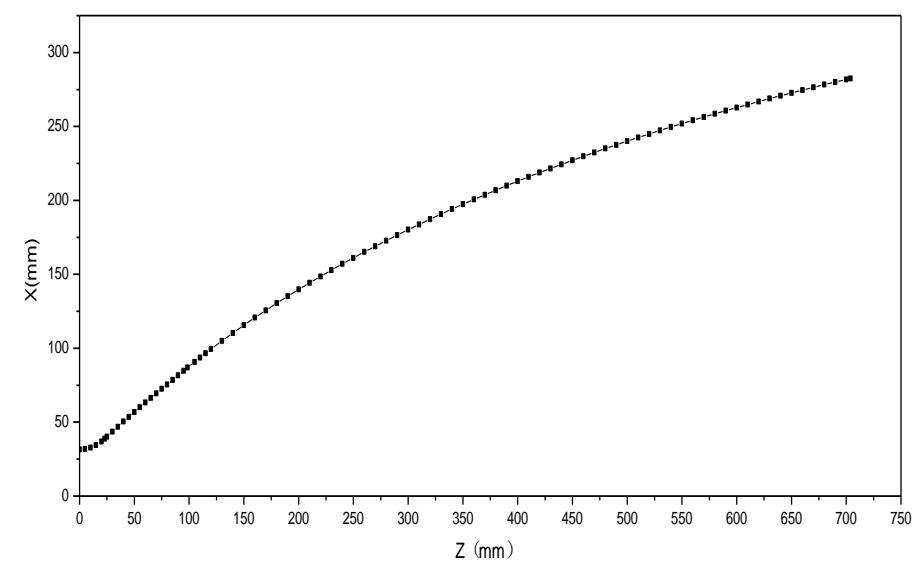

Figure 1. The inner moulding surface of the nozzle

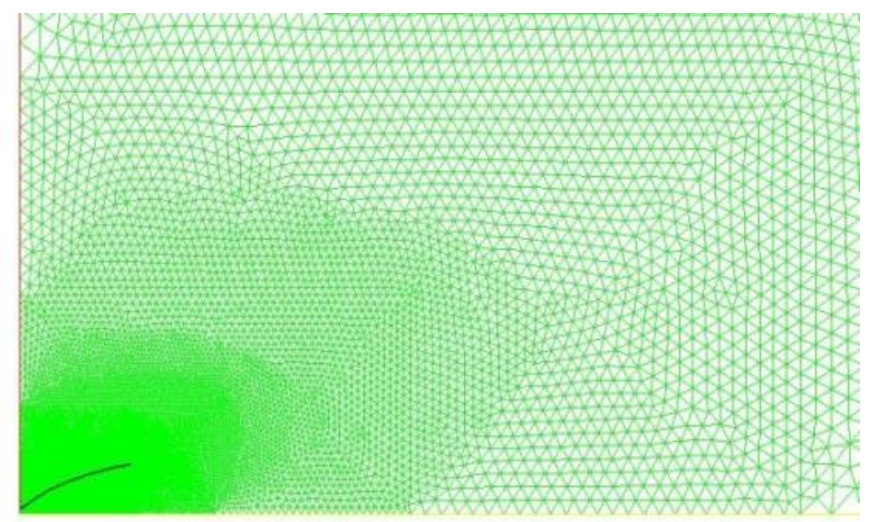

Figure 2. The computing domain of the plum

Figures 3-9 show the contour results of the Mach number distribution, the pressure distribution, the velocity distribution, the density distribution, the temperature distribution, and the streamline distribution of the plume, respectively. 


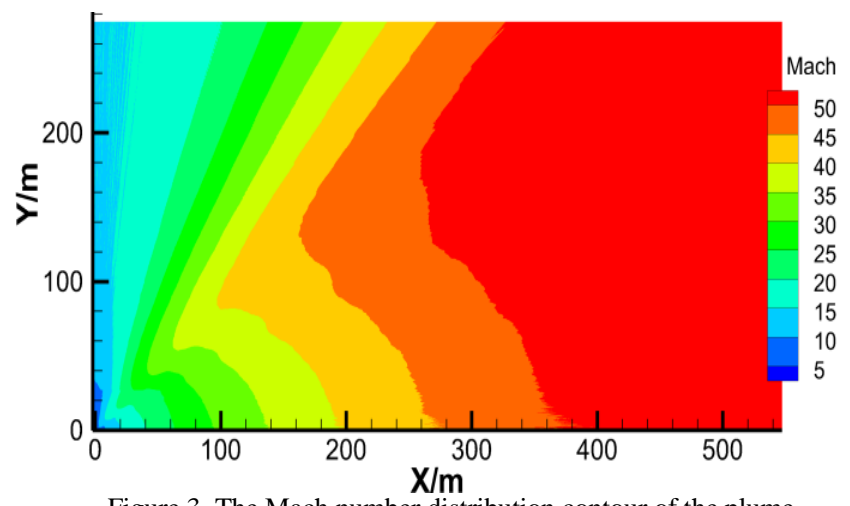

Figure 3. The Mach number distribution contour of the plume

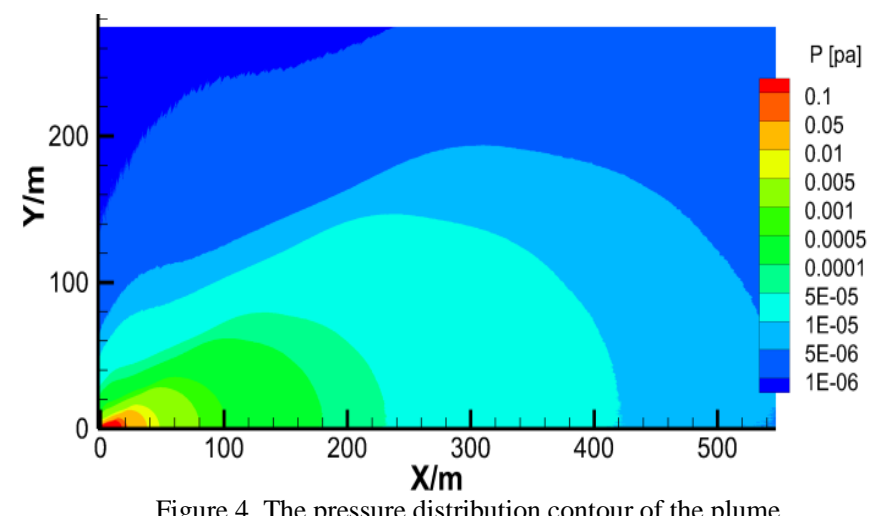

Figure 4. The pressure distribution contour of the plume

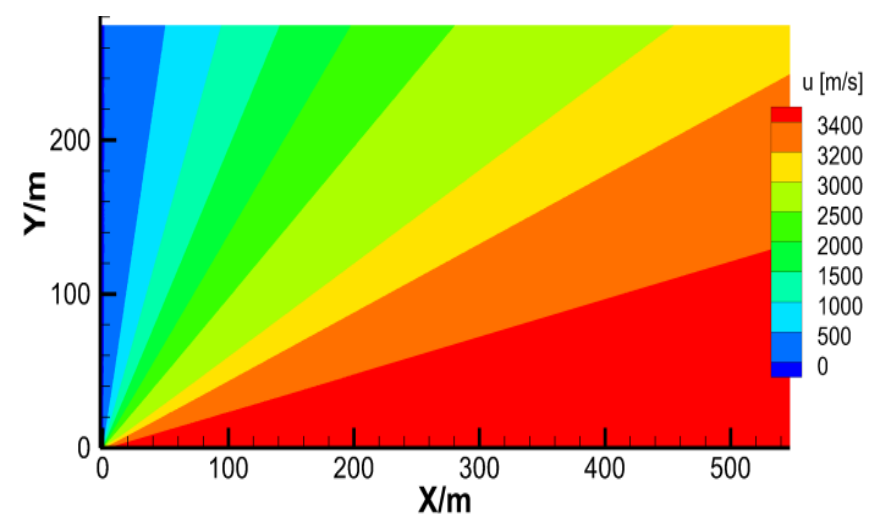

Figure 5. The axial velocity distribution contour of the plume

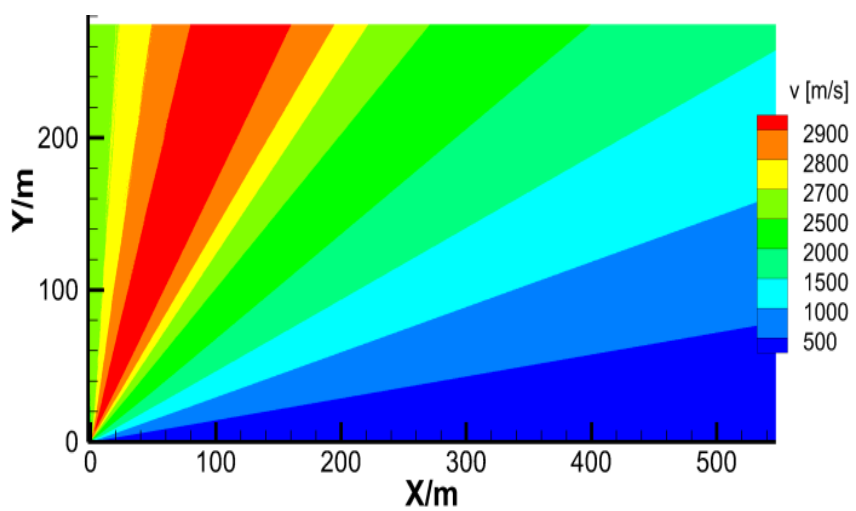

Figure 6 . The radial velocity distribution contour of the plume 


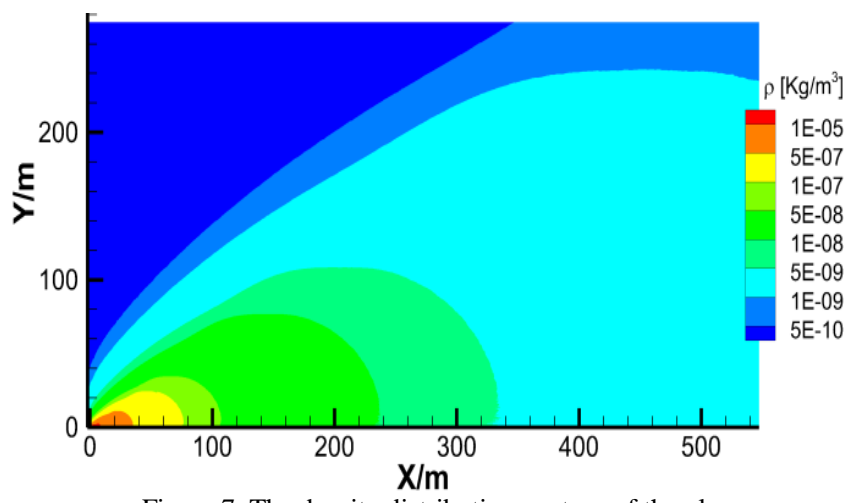

Figure 7. The density distribution contour of the plume

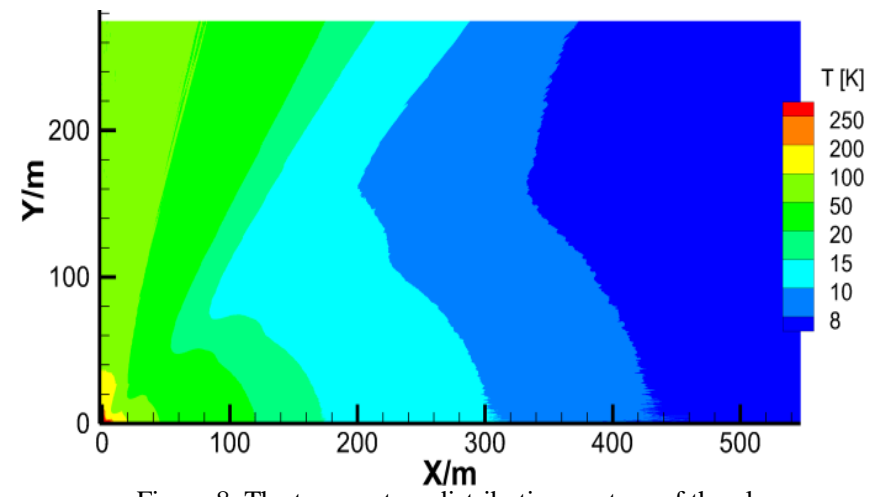

Figure 8 . The temperature distribution contour of the plume

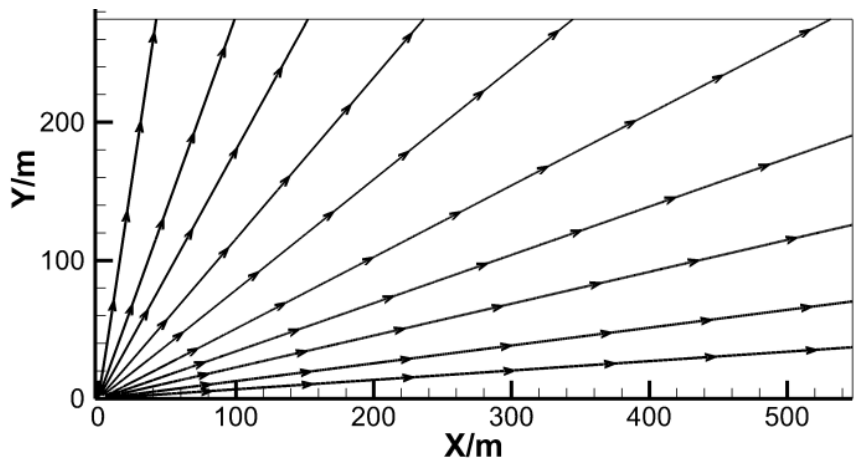

Figure 9. The streamline distribution contour of the plume

Step 2 According to the local Knudsen number $(K n)$, the boundary between the continuum flow and the rarefied flow is determined, and then this boundary would be the particle inlet of the DSMC model for simulation of rarefied flow.

The Knudsen number is defined as the ratio of the gas molecular mean-free path to the characteristic length scale: $K n=\lambda / l$, where $\lambda$ is the gas molecular mean-free path given by $\lambda=m /\left(\sqrt{2} \pi d^{2} \rho\right)$ and $l$ is the characteristic length scale (the nozzle outlet diameter in this article), $m$ is the molecular mass, $d$ is the molecular diameter, and $\rho$ is the flow density.

The flow region criterion put forward by Qian is taken in this paper:

- $K n<0.01$

- $0.01<K n<0.1$

no-slip continuum flow region

- $0.1<K n<10$

slip flow region

- $K n>10$

transition flow region

free molecule flow region

In this paper, $0.1 \leq K n$ is used as the criterion for determining the boundary between the continuum flow and the rarefied flow. 
Step 3 In the rarefied flow, the DSMC model of molecular dynamics is used to compute the rarefied plume and the reversed flow. Then, according to the different typical positions at the base of the new upper-stage, the convective heat flux is computed respectively.

The DSMC method put forward by Bird is now widely applied in engineering [15]. It is based on physics practice and uses a large number of simulation particles to represent numerous real gas atoms or molecules. It is a numerical method that employs the concepts of probability theory and statistics to analyse non-continuum gas flows. The simulation particles' motion, collision, and energy transmission are controlled by the probability theory, and during each time step, the changes of the simulation particles' position coordinates, velocity, and inner energy are recorded. Finally the macroparameters of rarefied flow are obtained through the statistic of the simulation particles' motion behaviours in each cell.

In this paper, the hypothesis of DSMC computation was made as follows: a. the particle collision is binary collision; $b$. the rotation energy of the molecule is considered, whereas the vibration energy and the chemical non-equilibrium effect are ignored; c. the plume is steady flow, and the initial incoming velocity of the sampling molecule is randomly drawn according to the Maxwell velocity distribution pattern; $d$. the particles are seen as variable-diameter hard spheres.

Through Steps 1 and 2, the inlet boundary of the DSMC model is determined. It is an annulus surface of the nozzle outlet $(0.295 \mathrm{~m} \leqslant R \leqslant 0.32 \mathrm{~m})$ and a cone surface whose coning angle to axis of nozzle is $65^{\circ}$. The simulation particles include five components of combustion gas, which are $\mathrm{N}_{2}, \mathrm{H}_{2} \mathrm{O}, \mathrm{CO}, \mathrm{CO}_{2}$, and $\mathrm{H}_{2}$. The DSMC computing domain is shown in Figure 10. The boundary conditions are set as follows: a. $Z=0 \mathrm{~m}, X=1.8 \mathrm{~m}$, and $Y=1.8 \mathrm{~m}$ are vacuum boundaries, some simulation particles going through which would be considered as escaping from the plume flow; b. $X=0 \mathrm{~m}$ and $Y=0 \mathrm{~m}$ are symmetry boundaries, some simulation particles arriving at which would have mirror reflections; c. the other boundaries including the nozzle and the base surfaces of the new upper-stage all are scattered reflection boundaries. If the DSMC method is used for the simulation of rarefied gas flow, the space step should be smaller than the molecular mean-free path, and the time step should be smaller than the local mean time of molecular collision. In this study, to enhance the computing efficiency, the space step is meshed by the geometric progression method, and the time step is represented as $\Delta \tau=3 \times 10^{-7} \mathrm{~s}$. The meshes are shown in Figure 10. The technological process of DSMC is shown in Figure 11, including the initialization, simulation of particles, boundary setting, adjustment of particles' serial numbers, computation of collision, and statistics of macroscopic flow characteristics.

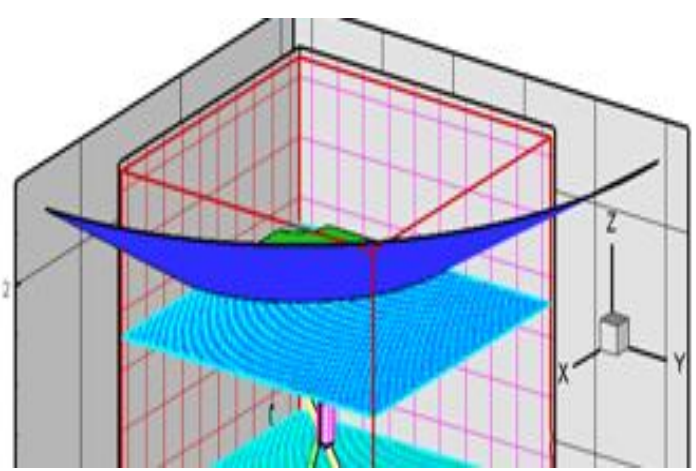

Figure 10. The DSMC model domain and meshes

\subsection{Radiative Heating of Plume and Nozzle}

The engine nozzle of the new upper-stage is cooled by the regenerative system and the radiative way. The radiative-cooling nozzle body is the majority, and its temperature is $1450 \mathrm{~K}$. The typical positions at the base of the upper-stage are heated by the plume convection, the plume radiation, and the nozzle radiation.

In this paper, the reversed Monte Carlo method (RMC) is proposed to calculate the plume radiation and the nozzle radiation at the same time, as shown in Figure 12. The simulation is taken through the following two steps: a) to reversely follow the trail of the beam and to solve all scattering events during the trail according to the radiation transmission reciprocity principle, the luminous beam is emitted from the radiation-received surface until the it is absorbed or escapes from the flow; b) the trail of the beam is followed from the beam-absorbed point again to the radiation-received surface, and during this process, the radiation from the beam-absorbed point to the radiation-received surface will be recorded. This method is very suitable for radiation computations, as the radiation-received surface is far smaller than the radiation source, by which the calculating efficiency can be increased. In this paper, the plume of the great thrust force engine expands 
extremely in the vacuum space and the scale of the plume and the high-temperature nozzle is greater than that of the typical positions' surface at the base of the upper-stage, so the RMC method is applicable.

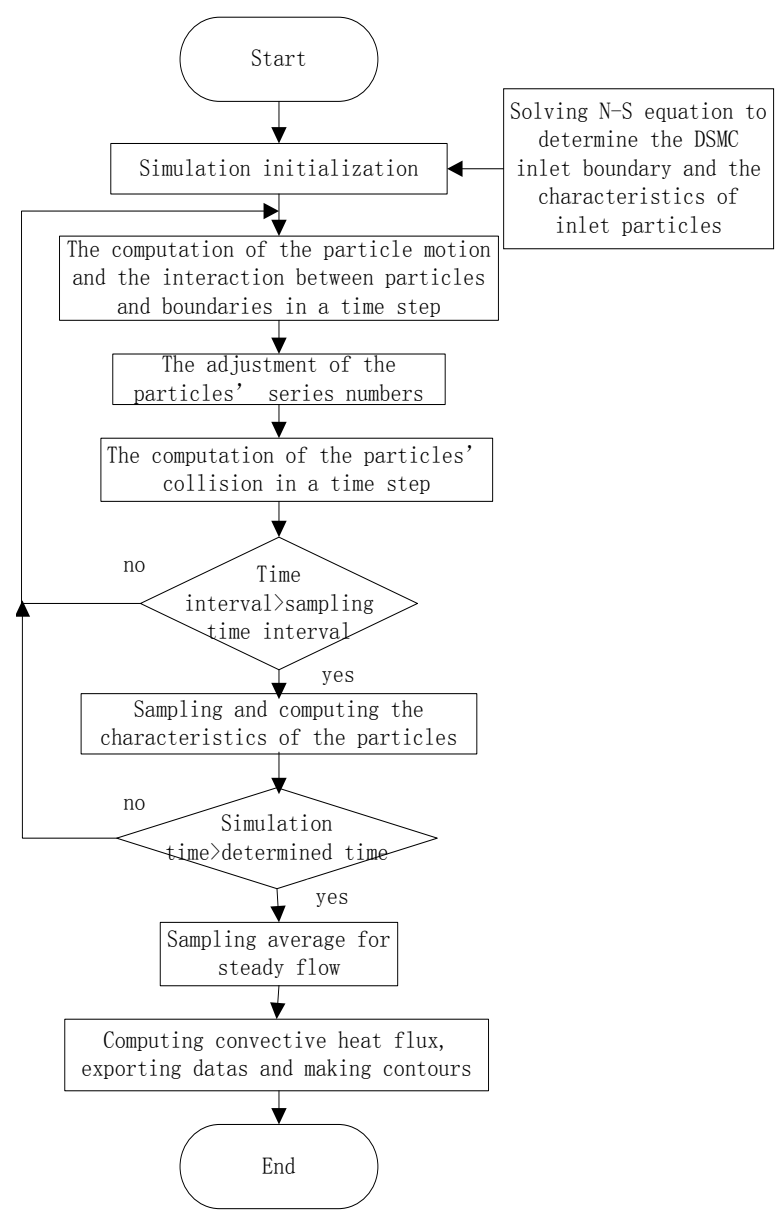

Figure 11. The technological process of DSMC

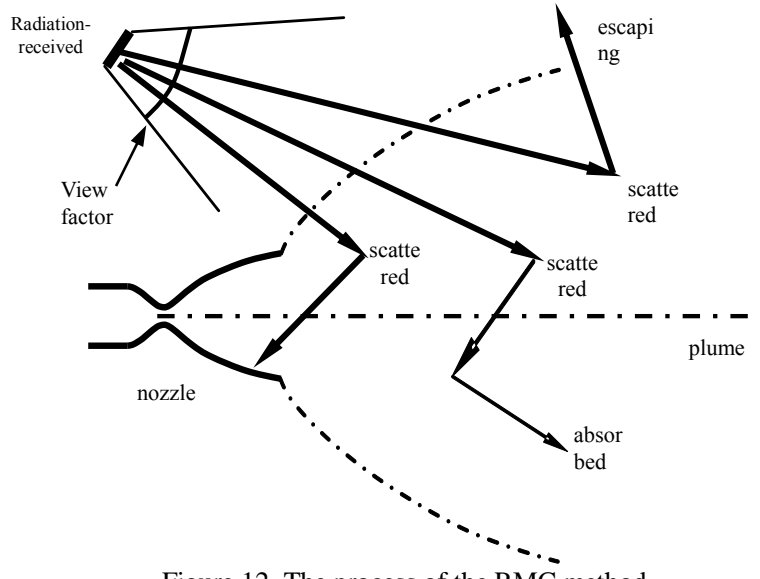

Figure 12. The process of the RMC method

To use the RMC method, the heat radiation meshed model should be established according to the flow characteristics, and at the same time the motion behaviours of the radiation beams and the plume boundaries should be modelled by mathematics equations. In this paper, a three-dimensional cylinder radiation model is employed. The inner meshes of the cylinder are shown in Figure 13. The engine nozzle is modelled according to the wall outline, and the wall temperatures are respectively given for the different bodies cooled by different ways. The gas radiation belongs to the medium radiation, which has the intensive spectral selection character and the capacity character. In this paper, nitrogen tetroxide $\left(\mathrm{N}_{2} \mathrm{O}_{4}\right)$ and dimethylhydrazine (UDMH) are used as propellants. The heat radiations of the combustion gas are mainly distributed in the 
infrared spectral coverage, which for different components is especially strong at different special wavelength, such as $2.7 \mu \mathrm{m}$ and $6.3 \mu \mathrm{m}$ for $\mathrm{H}_{2} \mathrm{O}, 4.3 \mu \mathrm{m}$ for $\mathrm{CO}$, and $4.7 \mu \mathrm{m}$ for $\mathrm{CO}_{2}$. These three components are considered in this study. The narrow spectral band model is employed, the computing wavelength scope is $2 \sim 200 \mu \mathrm{m}$, the total number of wave bands is 178 , the collision broadening effect and the Doppler broadening effect of the spectral beam are considered, and thus the mean absorbing coefficient of the gas component in the narrow spectral band is obtained. In this paper, Fortran is used to establish the RMC computing code.

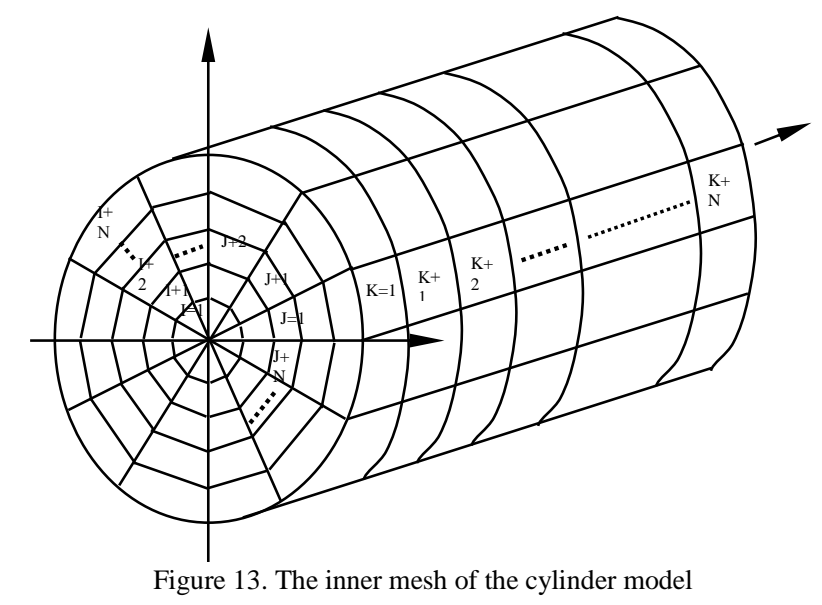

\section{Results and Discussions}

For the new upper-stage, the total heat fluxes of the different typical positions at the base are a very important design basis for the new thermal protection system, which includes three kinds of heat fluxes: the convective heat flux of the plume, the radiative heat flux of the plume, and the radiative heat flux of the nozzle. The results are listed in Table 1, and the typical positions are shown in Figure 14.

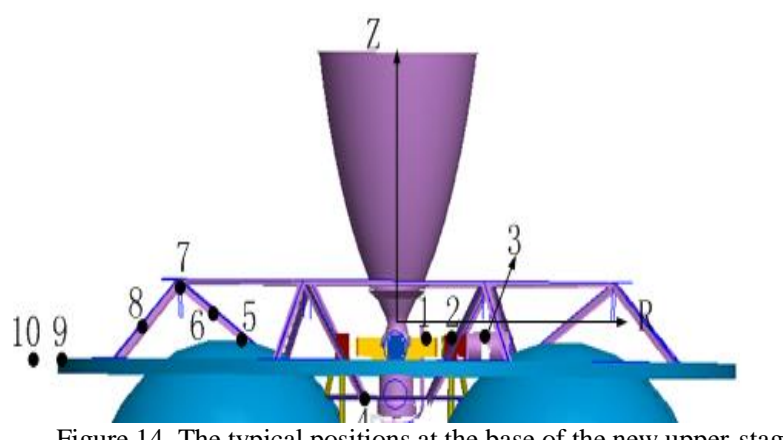

Figure 14. The typical positions at the base of the new upper-stage

During the working time in space, the nozzle of the engine needs to swing, and the swing angle is $\pm 25^{\circ}$, so the base heating environment of the swing engine is also calculated using the abovementioned method. The results are compared and listed in Table 1. It can be seen that the heat fluxes are greatly influenced for most typical positions except numbers 8,9 , and 10 by the swing engine. When the engine nozzle swing approaches some typical positions, the plume convective heat fluxes of these positions become greater, 1-2 times the results seen in Table 1, and the nozzle radiative heat fluxes become $20 \%$ greater than the results in Table 1 . The plume radiative heat fluxes do not change much.

\section{Evaluations}

In the 1970s, many theoretical analyses and ground wind tunnel experiments were carried out on the thermal environment at the bottom of the take-off stage of the American TITAN III rocket. The thermal radiation and convective heating of the single-nozzle and the multi-nozzle state were investigated. Kramer compared the results of theoretical analysis with those of ground experiments conducted in the Arnold Center for Engineering and Development. The theoretical model was verified and optimized by these ground experiments $[13,16]$, the cost of which were very expensive. 
Table 1 . The heat fluxes results of the typical positions

\begin{tabular}{|c|c|c|c|c|}
\hline \multirow{2}{*}{$\begin{array}{c}\text { Serial } \\
\text { number of } \\
\text { the typical } \\
\text { position }\end{array}$} & $\begin{array}{c}\text { Radiative } \\
\text { heat flux of } \\
\text { the nozzle }\end{array}$ & $\begin{array}{c}\text { Radiative } \\
\text { heat flux of } \\
\text { the plume }\end{array}$ & $\begin{array}{c}\text { convective } \\
\text { heat flux of } \\
\text { the plume }\end{array}$ & $\begin{array}{c}\text { Total heat } \\
\text { flux }\end{array}$ \\
\hline 1 & 10.9 & 0.224 & 0.01 & 11.13 \\
\hline 2 & 15.6 & 0.162 & 0.21 & 15.97 \\
\hline 3 & 17.7 & 0.155 & 0.39 & 18.25 \\
\hline 4 & 9.8 & 0.172 & 0.50 & 10.47 \\
\hline 5 & 12.96 & 0.182 & 1.14 & 14.28 \\
\hline 6 & 12.87 & 0.157 & 1.38 & 14.40 \\
\hline 7 & 8.7 & 0.193 & 0.47 & 9.36 \\
\hline 8 & 12.04 & 0.144 & 0.24 & 12.42 \\
\hline 9 & 5.76 & 0.312 & 0.95 & 7.02 \\
\hline 10 & 4.82 & 0.232 & 1.09 & 6.14 \\
\hline
\end{tabular}

Since the 1980s, with the development of computer technology, the numerical simulation of rocket motor plume has been used more and more widely. Flight experiments that replaced the ground test have been usually used for verification. The US space shuttle began its maiden voyage in 1981, ended it in 1984, and has completed five missions since then. NASA has done a great deal of work to measure the thermal environment of the shuttle bottom and compare the measured data with the predicted data to verify the simulation model [17-19].

In this paper, the flight experiment is carried out to evaluate the numerical simulation method. The base heating environment of the third stage of a flight launch vehicle is predicted by the method of this paper. The engine can also swing, and the swing angle is $\pm 10^{\circ}$ different from that of the engine in this paper. The propellants are the same, and the thrust force is $40 \mathrm{kN}$. The base structure is broader than that of the new upper-stage. In the flight experiment, two heat flux sensors are laid respectively at two typical positions at the base, and the normal of sensing face is parallel to the axis of the nozzle. The flight remote measured data curves are shown in Figures 15-16, and the measured data curves fluctuate because of the engine's swing. The comparison of the computed data and the measured data is shown in Table 2, and the position of the number 2 sensor is closer to the nozzle. It can be seen that the predicted data is in good agreement with the measured data. The simulation method in this paper is verified by this flight demonstration. It proves the validity of the numerical simulation method in this paper.

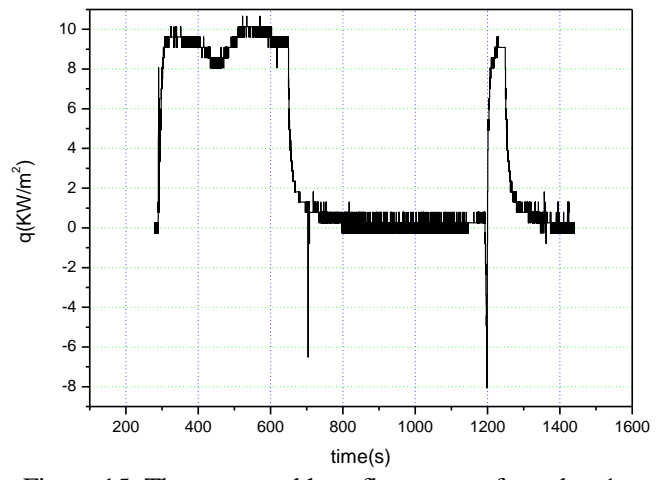

Figure 15. The measured heat flux curve of number 1 sensor

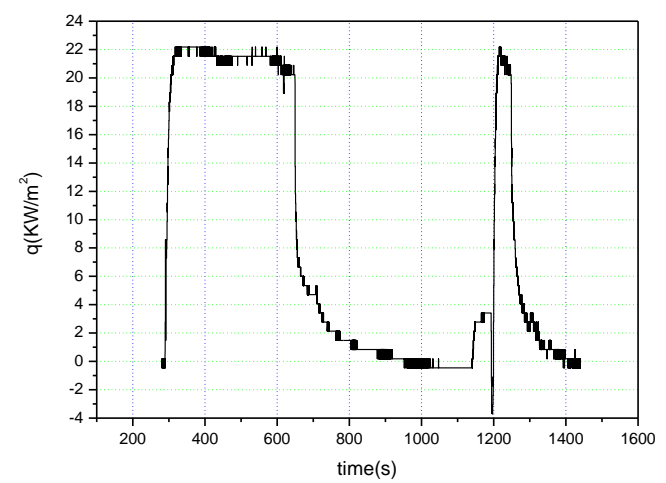

Figure 16. The measured heat flux curve of number 2 sensor

Table 2. The computed total heat flux and the measured total heat flux

\begin{tabular}{|c|c|c|c|}
\hline $\begin{array}{c}\text { Serial number } \\
\text { of the heat } \\
\text { flux sensor }\end{array}$ & $\begin{array}{c}\text { the computed total } \\
\text { heat flux } \\
\left(\mathrm{kW} / \mathrm{m}^{2}\right)\end{array}$ & $\begin{array}{c}\text { the measured total heat } \\
\text { flux } \\
\left(\mathrm{kW} / \mathrm{m}^{2}\right)\end{array}$ & $\begin{array}{c}\text { Percentage } \\
\text { error }\end{array}$ \\
\hline 1 & 11.6 & $5.6 \sim 10.1$ & $14.9 \%$ \\
\hline 2 & 22.6 & $16.7 \sim 22.3$ & $1.3 \%$ \\
\hline
\end{tabular}

\section{Conclusions and Future Work}

For the numerical analysis of base-heating for the new upper-stage of launch vehicles with great thrust force engines working in vacuum space, this scheme solves the N-S equations through a finite difference way coupled DSMC method of gaseous mixtures to obtain the convective heat flux of the plume. The RMC method is used to obtain the radiative heat flux of the plume and the nozzle. This scheme increases computing efficiency and achieves high accuracy in flight 
demonstration, so the results obtained in this paper are reliable for designing a new thermal protection system of the new upper-stage. On the basis of this elaborate base heating prediction, the weaknesses of the thermal protection can be found and the elaborate thermal protection can be realized, so the reliability of base heating prediction and thermal protection methodology can be increased. Some conclusions are drawn as follows:

- The velocity of the plume expansion in vacuum space is very high, and the majority of plume is rarefied flow. The plum radiative heat flux is low, as is the density and the pressure of the reversed flow. The plume convective heat flux is also not too great.

- The nozzle radiation effect on the base of the new upper-stage is an important factor for thermal protection designing.

- The thermal environment of the swing engine is very different from that of the non-swing engine, and the numerical simulation of and experiments on swing engine base-heating will be deeply studied in future work.

\section{References}

1. P. Y. Wang, H. F. Lu, and H. E. Cheng, "Dsmc Simulation of Plume Backflow from Bipropellant Attitude Control Engine," Journal of Engineering Thermophysics, Vol. 25, No. 4, pp. 640-642, 2004

2. J. Li, L. Yin, and L. Yan, "Two-Way Coupled Model for Rarefied Multiphase Flow,"Journal of National University of Defense Technology, Vol. 31, No. 3, pp. 6-10, 2009

3. Z. Y. Tang, B. J. He, and G. B. Cai, "Investigation on Boundary Conditions in Decoupled N-S/DSMC Method for Vaccum Plume Simulation of Thrusters," Journal of Propulsion Technology, Vol. 35, No. 7, pp. 897-904, 2014

4. Z. H. Li, H. Z. Zhong, A. P. Peng, and J. L. Wu, "A Numerical Method for Simulation Rarefied Two Phase Flow," Acta Aerodynamica Sinica, Vol. 33, No. 2, pp. 266-271, 2015

5. Y. F. Ding, Y. Zhao, and P. Y. Wang, "Simulation of High Altitude Solid Rocket Plume based on DSMC Method," Aerospace Shanghai, Vol. 34, No. 5, pp. 110-116, 2017

6. S. K. Dong, Y. Shuai, H. P. Tan, and L. H. Liu, "Computation of Radiative Heat Transfer in Participating Media using Backward Monte Carlo Method," Journal of Harbin Institute of Technology, Vol. 36, No. 12, pp. 1602-1604, 2004

7. X. Q. Qi, P. Y. Wang, J. Z. Zhang, Y. Shan, and H. E. Cheng, "Reverse Monte Carb Simulation on Infrared of Lobed Nozzle Mixer Plume," Journal of Shanghai Jiaotong University, Vol. 39, No. 8, pp. 1229-1232, 2005

8. F. Yang, P. Y. Wang, Y. Y. Bao, and P. Li, "Numerical Simulation on Secondary Launcher Exhaust Plume Base Heating," Aerospace Shanghai, No. 5, pp. 56-51, 2009

9. J. Y. Li, S. K. Dong, and H. P. Tan, "Influences of Phase Transition and Carbon Soot Impurity on the Radiative Properties of Alumina Particle Flow," Journal of Solid Rocket Technology, Vol. 35, No. 4, pp. 485-489, 2012

10. P. J. Sun, D. B. Wang, F. Yang, Y. R. Zhou, Y. Y. Bao, W. D. Zhang, et al., "Numerical Simulation and Flight Test Validation of a Launch Vehicle Altitude Engine Exhaust Plume Base Heating," Aerospace Shanghai, Vol. 33, No. S1, pp. 23-28, 2016

11. I. D. Boyd, P. F. Penko, and D. L. Meissner, "Numerical and Experimental Investigations of Rarefied Nozzle and Plume Flows of Nitrogen," in Proceedings of the $26^{\text {th }}$ Thermophysics Conference, pp. 91-1363, 1991

12. I. D. Boyd, P. F. Penko, D. L. Meissner, and K. J. DeWitt, "Experimental and Numerical Investigations of Low-Density Nozzle and Plume Flows of Nitrogen," AIAA Journal, Vol. 30, No. 10, pp. 2453-2460, 1992

13. O. Kramer, "Evaluation of Thermal Radiation from the TITAN III Solid Rocket Motor Exhaust Plumes," in Proceedings of the 5th Thermophysics Conference, Los Angeles, California, pp. 1-9, 1970

14. J. D. George and I. D. Boyd, "Simulation of Nozzle Plume Flows using a Combined CFD-DSMC Approach," AIAA, pp. 993454,1999

15. G. A. Bird, "Molecular Gas Dynamics and the Direct Simulation of Gas flows," Clarendon Press, 2003

16. O. Kramer, "TITAN III Convective Base Heating from Solid Rocket Motor Exhaust Plumes, " in Proceedings of AIAA/SAE 8th Joint Propulsion Specialist Conference, New Orleans, Louisiana, AIAA, pp. 1-7, 1972

17. T. F. Greenwood, "Development of Space Shuttle Base Heating Methodology and Comparison with Flight Data," in Proceedings of the JANNAF 13th Plume Technology Meeting, Chemical Propulsion Information Agency, CPIA-357, Vol. 1, pp. 67-82, Houston, Texas, April 1982

18. T. F. Greenwood, Y. C. Lee, R. L. Bender, and R. E Carter, "Space Shuttle Base Heating," Journal of Apacecraft and Rockets, Vol. 21, No. 4, pp. 339-345, 1984

19. T. F. Greenwood, D. C. Seymour, and R. L. Bender, "Base Heating Prediction Methodology used for the Space shuttle," in Proceedings of the JANNAF 10th Plume Technology Meeting, Chemical Propulsion Information Agency, CPIA-291, Vol. 2, pp. 253-312, SanDiego, California, December 1977 